\title{
Indicators for sustainable pedestrian mobility
}

\author{
S. Amoroso, F. Castelluccio \& L. Maritano \\ Department of Energy - Transport Section, University of Palermo, Italy
}

\begin{abstract}
Pedestrians walk everyday to satisfy their basic necessities. They need an environment that reflects their requirements and expectations, in other words "liveable" as much as possible. So we should offer tools that could allow a complete and clear evaluation of the existing walking environment, taking into account both pedestrian requirements and urban environment schemes. To measure pedestrian mobility performance we usually use indicators, which measure performances and grant a quick estimation of progress. Indicators are universally acknowledged synthetic and standard parameters that should help policy makers gauge whether things are going in the right direction or not. They should be outcome measures, rather than inputs, and they should capture broad results. The advantage of using indicators is to recognize the quality of urban mobility through economical and social view of an environment. The aim of this paper is to estimate and compare pedestrian mobility indicators, evaluating their value in relation to the quality of walking environment. It is important to analyze all the aspects that make it possible to clarify a definition of "sustainable pedestrian mobility". Their analysis has to be related to the individuation of a set of indicators that simultaneously take into account the users' needs and allow the complete comparison between different urban pedestrian mobility strategies policies. Variables such as visual attractiveness, comfort, system continuity, safety, security, accessibility and infrastructure quality of urban walking pathways are taken into account in our analysis. Finally, we analyze the generalized role that the proposed set of indicators could play in sustainable urban and transport development and planning, even in different economic social and urban contexts.
\end{abstract}

Keywords: pedestrians, indicators, urban walking environment. 


\section{Introduction}

The increasing car traffic undermines the quality of life into urban areas. More generally motorized traffic has grown so much that many important aspects of urban life are inhibited to such an extent that the question of sustainability has become an important topic. So the creation of a sustainable transport system has to be the main objective of European transport policy. This system should encourage facilities to connect all people and should be linked with economic, social and environmental aspects of society. Promoting walking and cycling (as an alternative to short car trips) is seen as the most important way to enact sustainability in more densely inhabited areas. Pedestrians and cyclists can provide the best opportunities of enhancing quality of life in urban areas if there are the conditions for a competition with the motorized means of transport. Often the best way to improve urban sustainability is to develop walking and cycling conditions and restrict automobile travel. Although this does not increase travel speeds and comfort, it improves liveability of urban context, with regard to environmental sustainability and affordability of the access to destinations.

Walking indeed can be considered the most basic form of transport, for the following reasons:

- It is universal. Virtually everybody walks, and all trips (with any mean of transport) include walking links;

- It is affordable. Economically and socially disadvantaged people tend to rely heavily on walking for transport. Walkability improvements provide equity benefits, and bear special costs associated with serving people with disabilities;

- It provides additional benefits, including exercise and enjoyment;

- Some walking facility improvements can be included in other transport budgets (e.g., transit facilities, airports, parking facilities, ferry terminals, etc.) because they serve these modes.

These points underline that "the healthiest and most sustainable modes of transport are walking (and cycling). Even car drivers become pedestrians to complete a trip, and effective public transport depends on people being able to walk comfortably to stations and stops." (SUSTRAN [1]).

So the role of walking can be considered as an important element to program a strategic growth of the city, in relation to its old and new areas. In most of the past visions the pedestrian was the measure of ideal urban spaces. This means a return to the original function of the existent infrastructure (streets and squares), primarily born for pedestrian use and only in a second time invaded by the ever more intense, fast and heavy vehicular traffic. "Indeed recent high-tech visions of relational spaces use the interaction of pedestrians as the basis for highly connected information environments" (Bazik [2]).

In addition to the target of a rebalanced mobility, the re-evaluation of walking can also means the creation of new poles of interest through which to reorganize the social life of the community and the city, especially in peripheral areas. The future walking and public space improvements should be implemented and established at the core of national and local transport, environment, health and 
social inclusion strategies, to make more incisive and large the benefits that will be harvest. The rapid growth of cities and the materialization of new expansion areas with unique functions (residential, industrial, commercial, etc.) resulted in the loss of the original integration in urban centres. They have lost more and more residential and social service functions, taking mainly a managerial and commercial connotation. So the redevelopment of neighbourhoods should also meet the need for a correct urban planning and to establish a set of poles of attraction, organized in a hierarchical system and designed with a dual function:

- to increase the provision of services and infrastructures displaced in decentralized locations (instead of, those still too focused);

- to organize and integrate these services and infrastructures, corresponding to different (but overall unitary) levels of aggregation with a correct and coordinated architectural design or requalification.

These poles should not only meet the basic needs of the people, expressed through a precise list of usage features and areas (standards), but also provide meeting places and re-aggregation points for urban communities, giving back to the city the functions that today are only a prerogative of the smaller towns. The expansion of cities is a problematic aspect, because residents and workers suffer its effects for their shifts, which reflect on the use of private car, so the mentioned poles could help to disadvantage commuter flows. Measures for real improvements should be an essential part of the overall strategy of sustainable urban development, so that unsustainable trends could not concealed by few cosmetic measures taken for walking. The future will take into account the good management of today in its different forms (environmental, economic, resource or space related): walking could provide the answer to sustainability. But to make desirable this form of mobility is important to meet the needs of potential users. So a specific literature review of theories of needs is requested.

\section{Theories of needs and pedestrian mobility}

The main aim of the identification of pedestrian needs is to provide a suitable and realistic ground to fulfill requirements for pedestrian facilities. So the identified needs could be used to construct indicators to determine in what measure urban background is suitable and attractive for pedestrians.

Maslow's hierarchy of needs (Maslow [3]) received a great attention and researchers have analyzed and evaluated each need in a multiplicity of scenarios. It is correct that some needs could take precedence over others (Alderfer [4]). For example the needs to satisfy thirst or to maintain a constant temperature are much more basic motivators than reaching an highest potential by engaging in self-actualization. But it is not clear how Maslow's hierarchy motivates behaviors in the exact hierarchical order. For example, it is not clear if a safety problem has always the priority on the thirst or another basic need. However it seems reasonable to think that behavior cannot be specifically predicted from levels of his hierarchy, particularly when those levels are near into the hierarchy. To face this problem, modern theorists, like Alderfer, modified the Maslow's hierarchy, giving to it an improved flexibility for explaining human behavior. 
"Existence, Relatedness and Growth" theory (Alderfer [4]) stated that these "growing" categories more appropriately describe human behavior. According to his theory, focusing exclusively on one need at a time will not effectively motivate changes in behavior. For example, a person would not select fulfill his thirst over seeking shelter during a thunderstorm. In addition, he simplified the structure of the hierarchy of needs levels (reducing the number of levels to three) and recognized differences in need preferences between different cultures, so the order of needs can be different for different people. User needs, according to Alderfer's theory, are subjective and vary for ability, age, lifestyle, culture, climate and many more contextual factors.

If applied to mobility the ERG assumes its three levels of needs considering any mode of transport. The first level deals with Existence issues, such the aspects that are related to safety, security, wait time and comfort. When we feel lost or unsure of how to proceed, it causes fear and uncertainty and certainly discomfort (Winters et al. [5]). The ease of way finding, or more generally, the usability of a particular form of transport (or transit system or bike facilities or pedestrian facilities) will also be included in this particular rung.

The second level of the hierarchy is connected to Relatedness needs. This rung includes belongingness needs (like the sense of belonging, loving and being loved, the sense of acceptance from others, etc.) and regards also self respect, achievement, high social status, positive recognition and a good reputation. Some of the principles regarding persuasion (such as consistency, reciprocity, authority, liking, etc.) are contained into this particular level.

The highest level of the hierarchy is Growth, which describes the innate desire for personal development, so it includes self actualization (the desire to realize ones highest potential and to seek things like truth, knowledge, peace, wisdom and justice).

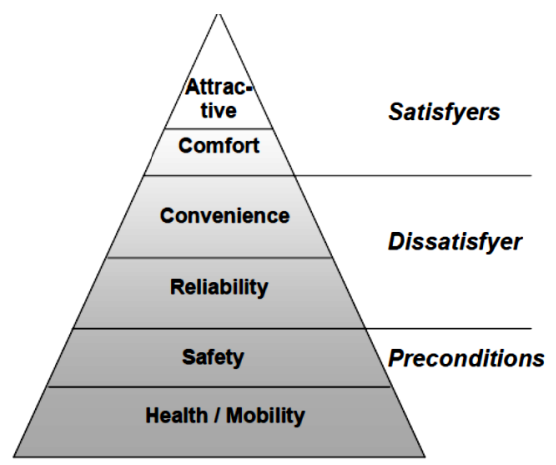

Figure 1: $\quad$ Pyramid of needs of pedestrians in public spaces (Methorst [6]).

Also Van Hagen's hierarchy proposed for categorize customer needs and based on Maslow's hierarchy (Van Hagen [7]) may be used to evaluate pedestrian needs. This model organizes hierarchically five levels of needs and 
presents them the basis of the walking decision-making process. His hierarchy can be used to understand the relative significance of the variables identified into pedestrian urban environment and it offers hypotheses on how the factors which affect peoples' decision to walk have to be considered, as we can see in figure 1 . The hierarchy of walking needs model alone does not explain the entire walking decision-making process. "The hierarchy of walking needs organizes the identified various urban form variables... ...into a hierarchy of prepotency" (Alfonzo [8]). Thus, some urban form variables are more fundamental (or necessary) within the decision-making process, it also includes the "feasibility", a nonurban form variable, as the most basic need, for which fulfillment is necessary to even consider urban form within the decision to walk. In fact, some needs are quite explicit and other are somewhat or totally hidden. This great variety will not make it easy to develop an effective and efficient strategy for the support and improvement of walking and sojourning. In the next paragraph the influence of population heterogeneity and composition on the urban and transportation planning is discussed.

\section{Pedestrian needs: uniformity and heterogeneity of categories}

The elements within the hierarchy have the role of basis for the walking decision-making process. It is the opportunity to fulfill these needs, however, that eventually may involve the decision to walk. With regard to walking behavior, people may differ on the basis of the opportunities they recognize: economic, social and cultural factors may all influence the relationship between the hierarchy of walking needs and a person's decision to walk. On the other side how much walkable is urban environment, in other words how much user needs are satisfiable, more it encourages pedestrians to the decision of walking. Surely basic pedestrian characteristics and skills are a basis for pedestrian quality needs:

- heterogeneity: almost every person is or can be a pedestrian and everyone has his own individual characteristics (abilities, needs, etc.) to be satisfied;

- $\quad$ the dimensions of the occupied space by a pedestrian are approximately 0,5 square meters, much less than other modes of transport;

- $\quad$ the walking average and maximum speed is slower than (almost) all other modes of transport;

- the action radius of a pedestrian is about 1 kilometre in Europe (less than other modes) and it depends on personal abilities;

- $\quad$ a pedestrian doesn't only walks, but he also sojourns in public space;

- a pedestrian is relatively vulnerable, so his safety and security have to be particularly focused.

With regard to heterogeneity, pedestrian needs are relate to a variety of personal characteristics, his or her social and physical environment and the availability of transport:

- $\quad$ lifestyle factors like age, gender, physical and mental abilities, education level, employment state, income, stage of life, family ties, social 
environment, culture, place of residence, vehicle ownership, availability of walking aids etc;

- climate and natural landscape features (factors like temperature, rain, humidity, wind and barriers, rivers, lakes, woods and scrubs, orography of terrain or the availability of flat land or walkable slopes, steep hills and mountains, etc.);

- $\quad$ land use characteristics (attraction points, facilities, degree of urbanism, the character of places of residence, relative locations of schools, work places, recreational facilities etc.).

- $\quad$ transportation characteristics (public transport availability and accessibility, pricing level and system etc.).

However (like true for any mode) the actual action radius depends on the urgency of the need and the reward one gets for going. Regarding the vulnerability aspect, a person needs to preserve his temperature, nutrition and activity, and particularly he needs to be protected from dangers, like too high or too low temperatures, accidents due natural or artificial causes (like motorized traffic, obstacles on his route, etc.). The efforts to survive translate into different long term needs for the various groups of pedestrians. So as individuals children for example, need to be enabled to grow up and acquire abilities to satisfy their mobility needs later on. Adults need to be able choose or create an environment where they can function as a human being and maintain their health. Adults need to have work, good working conditions, physical activity. The elderly and the handicapped need to be supported to be able to function in society. Thus, different stages of life induce different motives and needs for walking and sojourning in public space. On the lifestyle activity level preconditions for mobility, accessibility, safety, health and sustainability are laid down.

\section{Indicators of pedestrian urban sustainable mobility}

Urban and transportation planners have the fundamental task of addressing pedestrians' needs. For this reason the preliminary step is to knowing the composition of population that "use" the environment. As seen in the previous section, not everyone has the same needs, and needs can vary over time as well. One possible way for synthesizing of these needs is the use of indicators. Indicators quantify and simplify phenomena and help us understand complex realities, allow us to understand where we are, where we are going and how far we are from the goal. Indicators are meant to generate more effective decision processes, monitoring, transparency and communication, as well as more targeted planning and design. "Considering at the same time both the demand and offer perspective, they lead indeed the choice of the most advisable actions to take, technical and non-technical, when and where the users' requirements are not met, prioritize them and, as their name states in itself, indicate what to do in the process of change and, in particular, in organizing and designing a sustainable district. To this aim it is fundamental to measure the impacts that urban activities, in particular mobility" (Martincigh [9]). 
Another way of assessing and measuring walkability is to undertake a walking audit. An established and widely used walking audit tool is Pedestrian Environment Review System (PERS) which has been used extensively in the UK (Davies and Clark [10]). This system allows planners to evaluate the pedestrian environment upon five requirements of spaces and routes: convenience (routes should facilitate the desired journey without undue deviation or difficulty), connectivity (routes should link origins and destinations), conviviality (routes should be pleasant to use), coherence (routes should be continuous), conspicuity (the design should allow the user to be seen by, and to see, other pedestrians and vehicles to promote personal security and road safety). Another tool for the evaluation of walking environment is the Pedestrian Environmental Quality Index (PEQI), developed by the "San Francisco Department of Public Health" to assess the quality of the physical pedestrian environment and inform pedestrian planning needs. The PEQI draws on published research and work from numerous cities to assess how the physical environment impacts on whether people walk in a neighborhood. The PEQI is an observational survey which quantifies street and intersection factors empirically known to affect people's travel behaviors, and is organized into five categories: traffic, street design, land use, intersections, and safety. Within these categories are 30 indicators that reflect the quality of the built environment (that encourage walking while discouraging driving reduce traffic-related noise and air pollution) for pedestrians and comprise the survey used for data collection (Table 1).

Table 1: $\quad$ PEQI Indicators by pedestrian environmental category.

\begin{tabular}{|c|c|}
\hline INTERSECTION SAFETY & STREET DESIGN \\
\hline Crosswalks & Width of sidewalk \\
Ladder crosswalk & Sidewalk impediments \\
Countdown signal & Large sidewalk obstructions \\
Signal at intersection & Presence of curb \\
Crossing speed & Driveway cuts \\
Crosswalk scramble & Trees \\
No turn on red & Planters/gardens \\
Traffic calming features & Public seating \\
Additional signs for pedestrians & Presence of a buffer \\
\hline PERCEIVED SAFETY & TRAFFIC \\
\hline Illegal graffiti & Number of vehicle lanes \\
Litter & Two-way traffic \\
Lighting & Vehicle speed \\
Construction sites & Traffic volume \\
Abandoned buildings & Traffic calming features \\
\hline \multicolumn{2}{|c|}{ Public art/historic sites } \\
\hline \multicolumn{2}{|c|}{ Restaurant and retail use } \\
\hline
\end{tabular}


However, most common basic indicators for walkability audit are for example the number of inhabitants per age group (age classes, gender), the number of visitors that walk into the area, the estimated number of disabled persons, the number of one-person households and yet the distribution of household incomes, the vehicle ownership, the features of mobility (travel motives, number of trips, covered distances, etc.) and the culture regarding walking (public opinion, media statements).

Another synthetic indicator for evaluating walkability in the urban context is the Pedestrian Level of Service - LOS (Highway Capacity Manual, HCM [11]). The HCM calculates pedestrian LOS based on capacity and space requirements. Some communities measure pedestrian LOS and predict trips on feet that are associated with transit access. Qualitative attributes of pedestrian-friendly environments are described, but not quantified, in several sources. Pedestrian LOS evaluation criteria involve the provision of basic facilities, conflicts, amenities, motor vehicle LOS, maintenance, Transport Demand Management (TDM) and multimodal provisions. The first three pedestrian variables incorporate sub-elements. The pedestrian measures draw upon accepted facility design, signal timing, and other features supported in research. Pedestrian LOS ratings are defined by the measures of pedestrian safety features and the level of automobile-oriented development characteristics along the corridor. Moreover, the LOS ratings describe the basic level of disabilities acts compliance and the degree to which facility provisions encourage pedestrian use. Some authors proposed a statistical method for the calculation of Pedestrian LOS at crosswalks through a multi-variable regression analysis performed using the observed data of various types of intersections. Such a method evaluates the conditions of crosswalks at intersections as a function of pedestrian delay and the number of pedestrian-bicycle intersection. The limit of Pedestrian LOS model for crosswalk is that it provides a measure of a crosswalk's performance with respect only to pedestrians' safety and comfort, overlooking other fundamental aspects of walkability. These aspects just reflect the pedestrian's sphere of interaction within the walking environment.

However, indicators have become widely used in many different fields and play a useful role in highlighting problems, identifying trends, and contributing to the process of priority setting, policy formulation and evaluation and monitoring of progress. Most importantly, indicators can help to simplify a complex array of information concerning the health, environment and development nexus. In this respect, they are important for informing the public and decision-makers about key health and environmental problems, and actions required for their management. On the other hand, walkability indicators could represent a double-edge sword since their compliance does not exhaust the design of an integrated and harmonious environment in which all means of transport are considered.

In this perspective, urban and transport planning must shape a mobilityoriented environment where pedestrians play a main role. The next section deepens these remarks. 


\section{The role of indicators into sustainable urban planning}

The level of attention to be given to walking as a transport mode in tomorrow's cities has to be coordinated by the national policy but also initiated at a local level choosing interventions that could really favor the spread of this mode. So the chosen interventions are very important and their choice must be supported with the use of indicators that truly reflect the changing trends of the environmental variables of the studied system. To build truly useful indicators we have to consider that a sustainable environment includes characteristics and elements that should have meanwhile continuous and close communications with each other. These four characteristics are as follows:

- $\quad$ an hierarchy of open space: neighbourhood central square, pocket park with play, the local square, toddlers' greens, canal corridor;

- a vibrant mix of uses: mixed working areas, higher density housing and some working, predominantly residential areas.

- $\quad$ integrated transport systems: tram/light rail or main bus route, local bus route, neighbourhood street, local distributor, access road.

- good local facilities and amenities: shops, primary school, places of worship, community facilities such as pub, groceries, restaurants, shopping, coffee, banks etc.

"Overall support for the pedestrian environment," (Litman [12]) or walkability, becomes increasingly important as the world urbanizes and motorized modes constrain travel on feet on ever dedicated and confined spaces. This concern regards virtually every aspect of the pedestrian life. "Walkability takes into account the quality of pedestrian facilities, roadway conditions, land use patterns, community support, security, and comfort for walking" (Litman [12]). The interventions to implement pedestrian mobility may vary still in the same country. Although they may differ for size of the analyzed context (metropolis, city, town, suburb, etc.), always planning documents describe implementation frameworks, whose structures usually focus on similar models or methods, also for different transport modes. Always the individuation and measurement of the features that specifically characterize the analyzed modality and its application context (in terms of measurable and perceived elements or qualities), with a certain degree of objectivity by outside observers, are followed by the creation of (n) indexes. In fact using this methodology of study, one of the main obstacles to the achievement of a generally valid result is the nature of the collected data: some items therefore easily measurable (e.g. physical factors), while others are highly subjective and arbitrary (e.g. aesthetic and psychological factors). The second step is a validation procedure, often based on Stated Preferences surveys and other type of statistical analysis (like multi-variable regression analysis, etc.), assesses the real significance of the individuated variables, excluding those (n-m) indexes that do not reveal a real correspondence with the investigated environment. The next step shows the use of (m) validated indexes alone or to build more or less complex indicators. A further analysis on the validation of the results obtained by the indicators (in terms of concordance with the real trend of the studied environment) allows to assess the accuracy of 
the indicators and adaptability to the studied context. If the (p) built indicators do not fulfill the requested characteristics, the construction procedure is renewed adopting other indexes of the $(\mathrm{m})$ selected family. The cycle process continues with the application of policies to improve transport user's environmental characteristics and the successive re-measurement of selected features, as shown in figure 2. The objective is to aim the development of an organizational model of urban spaces that meets the needs of pedestrians in terms of safety, comfort and generally of their needs.

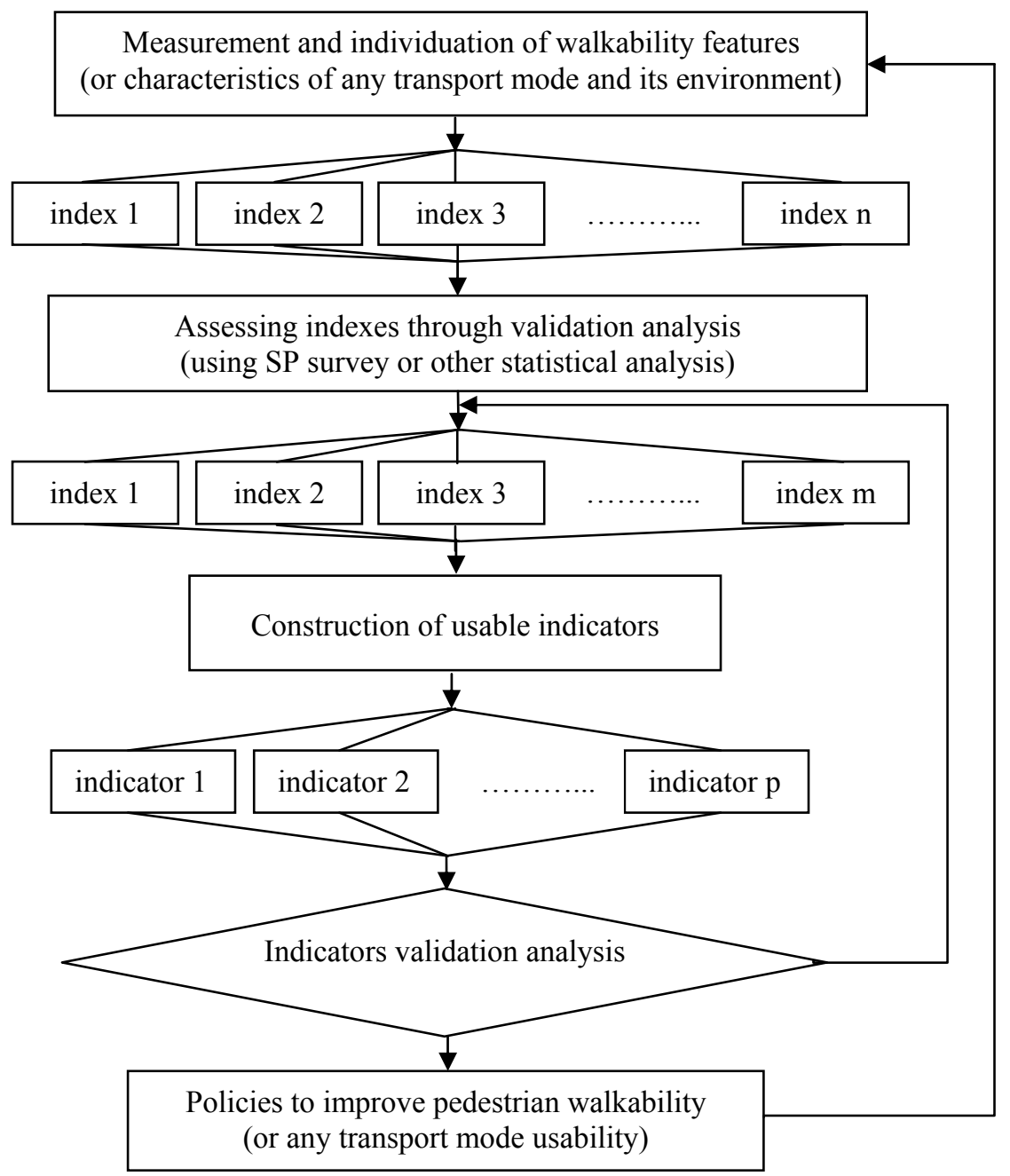

Figure 2: $\quad$ Flow chart on of sustainable urban policy process. 
The problem is that the variety of factors evaluative of the pedestrian space and their users' perception can intervene heavily on the accuracy and exhaustive nature of the remedial action of the contested issues. In a study devoted to the various characteristics deemed suitable for influencing physical activity (running feet) in the neighborhoods (Pikora et al. [13]) four basic categories of indicators were proposed.

They are related to functionality, the aesthetic, to practice (with close ties to the presence of tertiary distances acceptable), to safety. It should be that these categories of indicators, which are further broken down into elements and performance measures, as we see in figure 3.

\begin{tabular}{|c|c|c|c|c|}
\hline Categories & & Elements & & Sub-Elements \\
\hline \multirow{4}{*}{$\begin{array}{c}\text { Functional } \\
\text { Aspects }\end{array}$} & & $\begin{array}{l}\text { Supplied areas } \\
\text { for pedestrians }\end{array}$ & & $\begin{array}{l}\text { Path types; pavement types; infrastructure } \\
\text { maintenance; continuity of routes; }\end{array}$ \\
\hline & & Roads & & Cross section; \\
\hline & & Traffic & & Volume; speed; control instruments; \\
\hline & & Accessibility & (1) & $\begin{array}{l}\text { Roads and road-crossings design features; } \\
\text { distance between consecutive intersections; } \\
\text { other access points; }\end{array}$ \\
\hline \multirow{2}{*}{$\begin{array}{l}\text { Aesthetic } \\
\text { Aspects }\end{array}$} & & $\begin{array}{l}\text { Urban (road) } \\
\text { landscape }\end{array}$ & $\rightarrow$ & $\begin{array}{l}\text { Vegetation; gardens and roads maintenance; } \\
\text { environmental pollution; parking; cleaning; }\end{array}$ \\
\hline & & Visual aspects & & Architecture of points of interest; \\
\hline \multirow[b]{2}{*}{$\begin{array}{l}\text { Safety \& } \\
\text { Security } \\
\text { Aspects }\end{array}$} & & Personal & $\rightarrow 1$ & Paths illumination and surveillance; \\
\hline & & Traffic & & $\begin{array}{l}\text { Intersections and their support systems; } \\
\text { distance between motorized and pedestrian } \\
\text { traffic lanes; pathway signalization; }\end{array}$ \\
\hline $\begin{array}{l}\text { Practical } \\
\text { Aspects }\end{array}$ & & $\begin{array}{l}\text { Structures of } \\
\text { practical utility }\end{array}$ & & $\begin{array}{l}\text { Public and private amenities (parking, rest } \\
\text { areas, shops, etc.); }\end{array}$ \\
\hline
\end{tabular}

Figure 3: $\quad$ Public space features that influence the pedestrian traffic.

\section{Conclusions}

The concept of "walkability" can be expressed as the key element of an efficient urban transport system and includes all quality-connected aspects of urban space. In addition, the organization of a community attentive to the needs of pedestrians allows one to develop the transport system with the highest level of accessibility that can be planned, designed, implemented and managed. Many actions can be supported by the use of indicators and may provide improvements to be planned in several urban areas to enhance the above mentioned characteristics and elements (if integrated according to local needs). The increasing of the inclusive mobility could be reached providing a safe and suitable mobility and 
accessibility to the largest number of users (especially vulnerable ones), to the greatest number of places, building and maintaining high quality socially inclusive services and facilities. The planning and managing of pedestrian spaces should care of the correct design of paths (also cycle paths), recognizing that the roads are both a social space and a space for mobility. This must involve a balancing between the spaces devoted to cars and pedestrians, also creating a foot-dedicated environment to facilitate users' movement, social interaction, play and recreation. Furthermore, local authorities should meet pedestrians' needs ensuring a large number of infrastructures and amenities uniformly distributed throughout the city. Possible actions to be supported by the use of walkability indicators are the construction of a network of paths within the urban area, the provision of a public transport stops supply that meet all potential users needs, the design of sojourning or rest areas and intermodal nodes that ensure accessibility, information and safety. Safety of paths and crossings is also a basic feature for the attractiveness of walking. Limitation of motorized traffic flows is necessary to protect vulnerable users. So the use of safety/security-oriented indicators is significant to evaluate all utilizable measures $(30 \mathrm{~km} / \mathrm{h}$-zones within residential and commercial zones, over/underpasses, traffic lights crossings) to aim this goal. Also cyclist's role should be analyzed to not compromise safety of pedestrian over mixed pathways. Urban and transportation planners have the complex task of developing policies aiming maximize the opportunity of the urban environment enjoyment. So the use of indicators to design urban spaces, giving priority to soft mobility (walking and cycling) has a key role to promote walkability, consequently on modal rebalancing and, more generally, on economic, social and environmental aspects of urban life and mobility [14].

\section{References}

[1] SUSTRAN, Where next? Moving towards better urban transport, 1996.

[2] Bazik, D., A vision of public space: Relational space. Pedestrians quality needs, Final Report of the COST project 358, Walk 21, 2010.

[3] Maslow A., A theory of human motivation. Psychological Review, 1943.

[4] Alderfer, C.P., An empirical test of new theory of human needs. Organizational Behavior and Human Performance, 1985.

[5] Winters P. L., Perone J., Read M., Sankah I., Assessing Hierarchy of Needs in Levels of Service - Final Report, October 2005.

[6] Methorst R., Assessing Pedestrians' Needs, Final Report of the COST project 358, Walk 21, 2010.

[7] Van Hagen M., How to meet the needs of train passengers? A successful customer segmentation model for public transport. European Transport Conference - ETC. Netherlands, 6-8 October 2009.

[8] Alfonzo, M.A., To Walk or not to Walk? The hierarchy of Walking Needs. Environment and Behaviour, 2005.

[9] Martincigh L., Making transformation easier: the use of indicators, Final Report of the COST project 358, Walk 21, 2010. 
[10] Davies A. and Clark S., Identifying and prioritising walking investment through the PERS audit tool, Walk 21 Proceedings, 10th International Conference for Walking, New York, USA, October 2009.

[11] Transportation Research Board, Highway Capacity Manual, 2010.

[12] Litman, Todd., Economic Value of Walkability. Victoria Transport Policy Institute, BC, Canada, 2004.

[13] Pikora, T., Giles-Corti B., Bull F., Jamrozik K., Donovan R. Developing a framework for assessment of the environmental determinants of walking and cycling. Social Science and Medicine 56(8): 1693-703, 2003.

[14] Amoroso S., Caruso L., Castelluccio F. "Urban Indicators for sustainable mobility". WIT Transactions on Ecology and the Environment Series, Volume 148, 2012. 